\title{
Incorporating psychoeducation, mindfulness and self-compassion in a new programme for binge eating (BEfree): Exploring processes of change
}

Journal of Health Psychology I-14

(C) The Author(s) 2016

Reprints and permissions: sagepub.co.uk/journalsPermissions.nav DOI: $10.1177 / 1359105316676628$ hpq.sagepub.com

(S)SAGE

\author{
José Pinto-Gouveia', Sérgio A Carvalho', \\ Lara Palmeira', Paula Castilho', \\ Cristiana Duarte', Cláudia Ferreira', \\ Joana Duarte', Marina Cunha',2, \\ Marcela Matos' and Joana Costa'
}

\begin{abstract}
This study explores the efficacy of BEfree, a I2-session group intervention that integrates psychoeducation, mindfulness, compassion and value-based action, in a sample of overweight and obese women with binge eating disorder $(N=3 \mathrm{l})$. We used repeated measures analyses of variance and explored processes of change in binge eating and eating psychopathology. At post-intervention, participants decreased in binge eating severity, eating psychopathology, external shame, self-criticism, psychological inflexibility, body image cognitive fusion and increased self-compassion and engagement with valued actions. These results were maintained at 3- and 6-month follow-up. The changes in binge eating were mediated by the changes in the psychological processes promoted by BEfree.
\end{abstract}

\section{Keywords}

binge eating disorder, compassion, efficacy, mindfulness, processes of change

\section{Introduction}

The fifth edition of Diagnostic and Statistical Manual of Mental Disorders (DSM-V) introduced binge eating disorder (BED) as a formal diagnosis. BED is characterized by the consumption of a large amount of food ( $\geqslant$ once per week for the last 3 months), with a sense of lack of control and accompanied by at least three of the following symptoms: eating more quickly than usual, eating a large amount of food until feeling uncomfortably full, eating in the absence of hunger, secretive eating because of feelings of embarrassment, followed by marked distress and guilt after eating (American Psychiatric Association (APA), 2013).

\footnotetext{
'CINEICC, University of Coimbra, Portugal ${ }^{2}$ Instituto Superior Miguel Torga (ISMT), Portugal

Corresponding author:

Sérgio A Carvalho, University of Coimbra, CINEICC, Cognitive and Behavioural Centre for Research and Intervention, Rua do Colégio Novo, 3000-II5 Coimbra, Portugal.

Email: sergio.and.carvalho@gmail.com
} 
BED is present in 2 per cent-5per cent of community samples (e.g. Spitzer et al., 1993) and 20 per cent -40 per cent of people seeking weight loss treatments (e.g. Gormally et al., 1982; Spitzer et al., 1993). Moreover, individuals with BED are more likely to become obese earlier in life (Mussell et al., 1996) and present greater obesity severity (Picot and Lilenfeld, 2003). Additionally, obese individuals with BED are also more likely to have psychiatric comorbidities and medical problems when compared to those without BED (Bulik et al., 2002; Dingemans et al., 2002; Telch and Stice, 1998). In fact, research suggests that treating obesity in individuals with BED is more successful when BED is firstly addressed (e.g. Dingemans et al., 2002).

The development and maintenance of BED has been commonly conceptualized as the result of ineffective emotional regulation strategies (Leehr et al., 2015). Thus, binge eating can be viewed as an attempt to control negative internal experiences, such as difficult thoughts, painful emotions or urges perceived as uncontrollable (e.g. Lillis et al., 2011). This seems to be an inherent feature of psychological inflexibility, which is defined as rigid rule following and attempts to control difficult internal experiences, which leads to a decreased likelihood of engaging in value-based action (e.g. Hayes et al., 2006). Indeed, psychological inflexibility has been associated with disordered eating (Masuda et al., 2010, 2011) and binge eating (Duarte et al., 2015b; Duarte, Pinto-Gouveia and Ferreira, 2014). Additionally, a growing body of research has stressed the pervasive impact of shame (Duarte et al., 2014, 2015b) and self-criticism (Gilbert, 2002; Goss and Allan, 2009; Goss and Gilbert, 2002) on binge eating and eating psychopathology, which goes beyond the overall negative affect. Another characteristic that seems to be an important feature of binge eating conceptualization is the dysregulation of satiety and hunger awareness (Sysko et al., 2007) as well as the reactivity to food-related cues (Sobik et al., 2005).

In this line, contextual-behavioural approaches applied to eating disorders have emerged, in which the focus of interventions is not on reducing or changing internal negative experiences, but rather on promoting the change in the way one relates to those experiences. These approaches, such as the ones focused on mindfulness, self-compassion and promotion of values-based action, seem to be particularly useful for eating psychopathology and BED. It seems that promoting eating awareness through mindfulness improves eating regulation, enhances awareness of satiety and hunger cues and leads to an overall decrease in depression and anxiety in patients with BED (Kristeller and Wolever, 2010). In fact, recent research suggests that mindfulness interventions might impact on cortisol (e.g. O'Leary et al., 2015) and hypertension in females (Ahmadpanah et al., 2014). Additionally, interventions that target the reduction in shame and selfcriticism through the development of a more self-compassionate and reassuring stance towards one's difficulties and perceived flaws (e.g. Gilbert and Procter, 2006; Hermanto and Zuroff, 2016) seem to be useful in treating several psychopathological symptoms (see Leaviss and Uttley, 2015 for a review). Although similarly conceptualized, self-compassion is defined as the ability to be kind to oneself, to see one's difficulties as part of the common human experience and to be mindful of one's distress, as opposed to being self-judgemental, feeling isolated and getting over-identified with personal difficulties (Neff, 2003). Selfreassurance is more narrowly defined as the ability to be kind, caring and supportive when things go wrong (Gilbert, 2005, 2009, 2010). Selfcompassion seems to be positively linked to women's quality of life (Duarte et al., 2015a; Marta-Simões et al., 2016) and health-promoting behaviours (Dunne et al., 2016). Self-compassion also seems to be useful for BED treatment (Kelly and Carter, 2015; Kelly et al., 2014) and for dealing with body dissatisfaction in woman (Albertson et al., 2015). Furthermore, promoting acceptance and value-based action is associated with subjective well-being (Xu et al., 2014). Research shows that it can be useful when targeting weight and eating management (Juarascio et al., 2010, 2013; Lillis and Kendra, 2014) as well as for BED treatment (Masuda et al., 2014). Although approaches based on mindfulness, compassion and valuebased action seem to be useful individually for treating BED, attempts to integrate these potentially complementary approaches in theoretically 
coherent programmes for $\mathrm{BED}$ are to our knowledge inexistent. In fact, there seems to be a recent interest in integrating different approaches, such as acceptance-based and compassion-based approaches for medical conditions (Skinta et al., 2015), but none targeting BED.

Additionally, there is a general call for the study of mechanisms of the change underlying psychological interventions. It is of paramount importance to identify which processes promoted by an intervention are responsible for treatment-induced change (McCracken and Gutiérrez-Martínez, 2011; Murphy et al., 2009), in order to further develop parsimonious interventions and establish the connection between what is targeted and promoted in an intervention and the observed outcomes (Kazdin, 2007).

The goal of this study is twofold: (1) to explore the efficacy of BEfree - a psychological group programme for BED in overweight and obesity that integrates a psychoeducation component and new components aiming at developing mindfulness, self-compassion and promoting value-based action, at 3- and 6-month follow-up and (2) to explore which psychological processes mediate the therapeutic changes in binge eating and eating psychopathology at post-intervention. We expect that BEfree will be efficacious in reducing binge eating severity and eating psychopathology, as well as in diminishing maladaptive psychological processes (psychological inflexibility, body image cognitive fusion, selfcriticism, external shame) and in improving more useful ones (engage with valued living, mindfulness - particularly the ability to act with awareness, non-reacting and non-judging - and self-compassion). We also expect that the psychological processes promoted by BEfree will mediate the changes at post-intervention.

\section{Method}

\section{Participants}

This study was conducted in a sample of women $(N=31)$ between 18 and 55 years old who met criteria for BED and overweight or obesity (body mass index $(\mathrm{BMI}) \geqslant 25)$. Participants were excluded from treatment if they presented at least one of the following: (1) medical conditions that affect weight; (2) severe psychiatric problems (current severe major depressive episode, bipolar disorder, substance abuse and borderline personality disorder); (3) cognitive impairment or significant difficulties in understanding the contents and questionnaires; (4) medication intake that may cause the changes in weight or appetite or (5) unavailability to attend weekly sessions.

\section{Procedure}

Participants were recruited both at the Endocrinology Service $\quad(n=18 ; \quad 58.1 \%)$, Coimbra's University Hospital Centre (CHUC), and through advertisements in national newspapers $(n=13 ; 41.9 \%)$. BED diagnosis was carried out using Eating Disorders Examination (EDE) interview as well as resorting to the scores of Binge Eating Scale (BES) as a complimentary criterion (assuming BES $>17$ as the threshold for binge eating; Duarte et al., 2015b; Marcus et al., 1985). BMI was assessed using a body mass analyser (Tanita-SC-330) accurate to $0.1 \mathrm{~kg}$. Exclusion criteria were assessed through Structured Clinical Interview for DSM-IV Axis I Disorders (SCID-I) and Structured Clinical Interview for DSM-IV Axis II Disorders (SCID-II) interviews (using exclusively the sections that assess the exclusion criteria). All clinical interviews were conducted by clinical psychologists who were part of the research team and not responsible for delivering the intervention. In order to assess the changes as a result of the intervention, participants completed a battery of self-report questionnaires at pre-intervention, post-intervention, 3 and 6 months after the intervention. BED criteria were also assessed at these different time moments, through EDE interview (Figure 1).

\section{Measures}

BES (Duarte et al., 2015b; Gormally et al., 1982) comprises 16 items and is a self-report measure of binge eating symptomatology. Respondents are asked to choose the statements that best describe their experience. BES total 


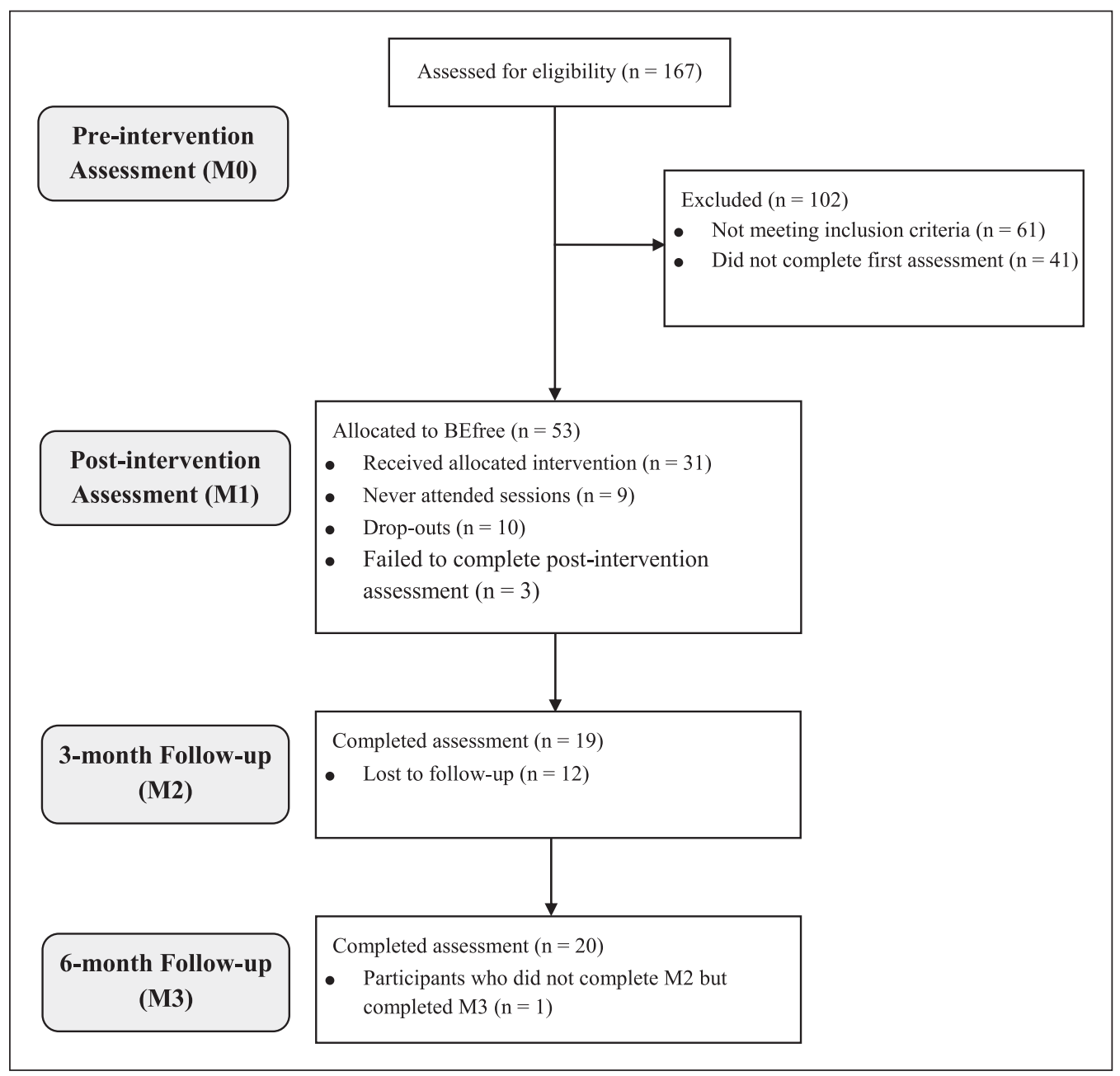

Figure I. Participants' enrolment diagram.

score ranges from 0 to 46 and higher scores reflect higher severity of binge eating symptoms. Both the original and Portuguese versions revealed good internal consistencies. This study presented an internal consistency of $\alpha=.85$.

EDE 16.0D (Fairburn et al., 2008; validation for Portuguese by Ferreira et al., in preparation) is a semi-structured clinical interview developed to assess the frequency and intensity of disordered eating behaviours and attitudes. Although comprising four subscales (restraint, eating concern, shape concern and weight concern), it provides a global score for overall eating psychopathological severity. The interview evaluates the presence of BED as well as general eating psychopathology. EDE has consistently demonstrated good psychometric properties (e.g. Fairburn et al., 2008) and in this study presented an internal consistency of $\alpha=.87$.

The Acceptance and Action Questionnaire-II (AAQ-II; Bond et al., 2011; translation and adaptation: Pinto-Gouveia et al., 2012) is a seven-item self-report measure of psychological inflexibility. This instrument assesses tendencies to make negative evaluations of private events and the unwillingness to be in contact with private events (e.g. 'I'm afraid of my feelings' and 'my painful memories prevent me 
from having a fulfilling life'). Respondents analyse how each statement applies to them, using a 7-point Likert response format type $(1=$ never true; 7=always true), and the total score is attained by summing the items, with higher results indicating higher levels of psychological inflexibility. The original version has good psychometric qualities, including an internal consistency of $\alpha=.84$ (Bond et al., 2011). The Portuguese version had an internal consistency of $\alpha=.89$ (Pinto-Gouveia et al., 2012). The results in this study found an internal consistency of $\alpha=.92$.

Cognitive Fusion Questionnaire-Body Image (CFQ-BI; Ferreira et al., 2015) is a selfreport questionnaire based on the original CFQ (Gillanders et al., 2014). It comprises 10 items that measure cognitive fusion related to body image. Participants are asked to rate the extent to which each statement (e.g. 'My thoughts relating to my body image cause me great distress or emotional pain') is true regarding their own experience, using a 7-point Likert scale $(1=$ Never true $; 7=$ Always true $)$. The original study presented good internal consistency, retest reliability, discriminant, convergent and divergent validities (Ferreira et al., 2015). This study found an internal consistency of $\alpha=.97$.

The Engage Living Scale (ELS; Trompetter et al., 2013) is a self-report measure developed to assess engagement with value-driven behaviour. The original version comprises 16 items which respondents should rate according to their personal experience on a 5-point Likert scale $(1=$ completely disagree; 5=completely agree). Higher scores express increased clarity and engagement with personal values and greater life fulfilment. Recently, a shorter nineitem version of ELS has been used, showing good internal consistency $(\alpha=.88$; Trindade et al., 2015). This study found similar internal consistency $(\alpha=.86)$.

Other as Shamer Scale (OAS; Goss et al., 1994; Matos et al., 2015) is 5-point Likert scale composed of 18 items that assess the perception of being negatively evaluated by others. Higher scores indicate higher external shame (Goss et al., 1994). OAS has consistently showed high internal consistency, both in clinical and nonclinical samples $(\alpha=.96$ and .92 , respectively; Goss et al., 1994). Similar results were found for the Portuguese version $(\alpha=.91$; Matos et al., $2015)$. This study found internal consistency of $\alpha=.96$.

Forms of Self-Criticising/Attacking \& SelfReassuring Scale (Gilbert et al., 2004) is a 22-item self-report measure of forms of self-criticism (inadequate-self, which focuses on a sense of personal inadequacy ('I am easily disappointed with myself'), and hated-self, which measures the desire to hurt or persecute the self ('I call myself names')) and the ability to self-reassure when things go wrong ('I find it easy to forgive myself'). Cronbach's alphas in non-clinical samples ranged from .89 to .91 for inadequate-self, .82 to .89 for hated-self and .82 to .88 for reassured-self. In clinical samples, Cronbach's alphas ranged from .87 to .89 for inadequate-self, .83 to .86 for hated-self and .85 to .87 for reassured-self. This study found internal consistencies of $\alpha=.91$ for inadequate-self, $\alpha=.67$ for hated-self and $\alpha=.93$ for reassured-self.

Self-Compassion Scale (SCS; Neff, 2003; Portuguese version by Castilho et al., 2015) is composed of 26 items distributed in six subscales: three positive (self-kindness, common humanity and mindfulness) and three negative (self-judgement, isolation and over-identification). Although the factor structure of SCS is an ongoing topic of research (López et al., 2015; Neff, 2016), it has been recently shown that SCS might also present a two-factor structure: one factor that assess self-compassion attitude (a composite of self-kindness, common humanity and mindfulness) and one factor of a selfcriticism attitude (that results from the sum of self-judgement, isolation and over-identification). Participants respond according to a 5-point Likert scale ( 1 = almost never; $5=$ almost always). Previous studies found adequate model fit and good internal consistency $(\alpha=.91$ for self-compassion and $\alpha=.89$ for self-criticism; Costa et al., 2015). This study presented good internal consistencies for both the self-compassion factor $(\alpha=.94)$ and the self-criticism factor $(\alpha=.91)$. 
Five Facet Mindfulness Questionnaire-15 (FFMQ-15, Baer et al., 2006, Portuguese version by Gregório et al., in preparation) is composed of 15 items and is the shorter version of the original 39 items questionnaire. FFMQ measures the dispositional and multifaceted mindfulness characteristics. Participants are asked to rate how mindful they feel in daily life in a 5-point Likert scale $(1=$ never or very rarely true; 5=very often or always true). FFMQ-15 presents the same five-factor structure as the original version, as well as good internal consistency (ranging from .65 to .86). In this study, the internal consistencies of the subscales were below the recommended: Observing $(\alpha=.26)$, Describing $(\alpha=.20)$, Act with awareness $(\alpha=.50)$, Non-judgement $(\alpha=.45)$ and NonReacting $(\alpha=.26)$. The total scale also presented a poor internal consistency $(\alpha=.37)$.

\section{The intervention: what is BEfree?}

BEfree is a psychological programme carried out in a group format that integrates a psychoeducation component with new contextual-behavioural approaches such as mindfulness, self-compassion and value-based committed action. It is composed of 12 sessions, 2 hours 30 each, and runs in small groups (minimum of 10 and maximum of 15 participants). In this study, sessions were carried out by three cognitive-behavioural clinical psychologists, who had previous training in contextual-behavioural therapies. All sessions were structured as follows: (1) an initial moment of sharing personal experience, (2) a 5-minute mindfulness exercise, (3) the session theme, (4) an eating mindfulness exercise and (5) summary of the session content and homework assignments. Psychoeducation sessions focused on the evolutionary foundations of emotions and binge eating as an emotional regulation strategy. Experiential exercises, such as loving-kindness, safe-place and compassionate image, were used to promote self-compassion and diminish selfcriticism and shame. Sessions focusing on mindfulness included different exercises, such as mindfulness breathing meditations, body scan, mindfulness of thoughts, as a way of promoting acceptance and decentring of internal difficult experiences. Finally, sessions focused on the values and committed action promoted clarification of participants' meaningful life directions, particularly related to health and self-to-self relating. This engagement with personal values is also the basis for establishing new goals, dealing with setbacks and preventing relapse.

\section{Statistical analyses}

All statistical procedures were conducted using IBM SPSS (version v.23). To test whether there were significant differences between pre- and post-intervention variables, paired sample $t$-tests were calculated. To explore whether the therapeutic gains were maintained at 3- and 6-month follow-up, repeated measures analyses of variance (ANOVAs) were carried out. A post hoc power analysis conducted using $G$ power showed that at a significance level of $p<.05$, with four different measurement moments and an effect size of $f=0.25$, the power analysis was 91 per cent. Post hoc analyses using Fisher's least significant difference (LSD) test were further computed to explore pairwise differences (post-to-3 months; post-to-6months). The effect sizes for the paired sample $t$-tests were calculated using Cohen's $d$, with 0.2 indicating a small effect, 0.5 a medium effect and 0.8 a large effect (Cohen, 1988).

In order to explore which psychological processes promoted by BEfree mediated the therapeutic changes in the outcome variables, we used the macro MEMORE for SPSS (Montoya and Hayes, 2016). This novel statistical procedure allows to test mediation effects in twocondition within-participants' designs. In these models, the mediator and the outcome are the calculated change between post- and pre-intervention, and the independent variable ' $\mathrm{X}$ ' is the mere passage of time from pre- to post-intervention, that is, the effect of the intervention. MEMORE generates percentile bootstrap confidence intervals (CIs) for inference about the indirect effect, based on 5000 bootstrap samples. A significant indirect effect occurs when the interval between the lower and upper bounds of CI does not include 0 . 
Table I. Mean, SD and effect size of the pre- to post-intervention differences in outcomes and process variables $(N=3 I)$.

\begin{tabular}{|c|c|c|c|c|c|c|c|}
\hline \multirow[t]{2}{*}{ Variables } & \multicolumn{2}{|c|}{ Pre-intervention } & \multicolumn{2}{|c|}{ Post-intervention } & \multirow[t]{2}{*}{$t$} & \multirow[t]{2}{*}{$p$} & \multirow[t]{2}{*}{$d$} \\
\hline & M & $S D$ & M & $S D$ & & & \\
\hline \multicolumn{8}{|l|}{ Outcome variables } \\
\hline Body mass index & 35.35 & 6.07 & 34.71 & 6.19 & 2.84 & .008 & 0.52 \\
\hline Binge eating & 28.66 & 8.04 & 11.66 & 8.62 & 10.49 & $<.001$ & 1.95 \\
\hline Eating psychopathology & 3.74 & 1.11 & 2.22 & 0.84 & 6.71 & $<.001$ & $\mathrm{I} .34$ \\
\hline \multicolumn{8}{|l|}{ Process variables } \\
\hline Psychological inflexibility & 30.60 & 8.85 & 25.43 & 8.47 & 3.84 & .001 & 0.70 \\
\hline Body image cognitive fusion & 43.14 & 15.82 & 31.21 & 13.44 & 4.53 & $<.001$ & 0.84 \\
\hline Engaged with valued living & 27.82 & 5.89 & 30.75 & 6.55 & -2.91 & .007 & 0.55 \\
\hline External shame & 32.53 & |7.6| & 24.63 & 16.59 & 3.80 & .001 & 0.69 \\
\hline Inadequate-self & 21.13 & 8.22 & 16.50 & 7.80 & 3.32 & .002 & 0.61 \\
\hline Hated-self & 6.24 & 3.95 & 4.07 & 3.65 & 2.71 & .011 & 0.50 \\
\hline Reassured-self & 14.25 & 7.32 & 16.18 & 7.47 & -2.05 & .05 & 0.39 \\
\hline Self-judgement & 10.15 & 2.29 & 8.13 & 2.07 & 2.74 & $<.001$ & 1.09 \\
\hline Self-compassion & 8.12 & 2.12 & 9.15 & 2.12 & -3.13 & .004 & 0.58 \\
\hline \multicolumn{8}{|l|}{ Mindfulness } \\
\hline Observe & 9.40 & 1.92 & 9.77 & 2.11 & -1.06 & .300 & 0.19 \\
\hline Describe & 8.62 & 1.99 & 9.48 & 3.12 & -1.71 & .098 & 0.32 \\
\hline Act awareness & 8.47 & 1.93 & 9.40 & 1.92 & -2.51 & .018 & 0.46 \\
\hline Non-judge & 8.60 & 1.98 & 10.73 & 2.15 & -3.35 & .002 & 0.61 \\
\hline Non-react & 8.90 & 1.63 & 9.07 & 2.03 & -2.50 & .019 & 0.46 \\
\hline Total & 43.24 & 4.43 & 48.59 & 7.61 & -3.85 & .001 & 0.71 \\
\hline
\end{tabular}

SD: standard deviation.

\section{Results}

\section{Sample's characteristics}

Participants who attended BEfree intervention were on average 39.68 years old (standard deviation $(S D)=10.29$ years) and had a mean of 14.93 $(S D=2.48)$ years of schooling. Concerning marital status, 63.3 per cent of participants were married and the majority had a medium socio-economic status (48.3\%). Participants had a mean BMI (kg/ $\left.\mathrm{h}^{2}\right)$ of $35.35(S D=6.07)$. Moreover, no differences were found between participants recruited at Endocrinology Service and through national newspapers in all variables in study.

\section{Pre- to post-intervention differences}

The results from paired sample $t$-tests are presented in Table 1. Participants showed a decrease in binge eating and eating psychopathology.
There was also a decrease in BMI after intervention, even though weight loss was not identified as BEfree's primary outcome. Regarding psychological processes promoted by BEfree, there was a significant decrease in psychological inflexibility, body image cognitive fusion, external shame and self-criticism (both in its toxic forms - inadequate and hated-self - and the more general self-judgement) and an increase in engagement with valued living, self-compassion, self-reassurance and mindfulness facets (acting with awareness, non-judging and nonreacting). Effect sizes were medium to large in both outcome and process measures.

\section{Durability of change: 3- and 6-month follow-up}

To explore the maintenance of therapeutic gains, we conducted repeated measures ANOVA 
Table 2. Means and SDs for the 3-month $(n=19)$ and 6-month $(n=20)$ follow-up assessments, and repeated measures ANOVA results for the comparison between post-intervention, 3- and 6-month follow-up.

\begin{tabular}{|c|c|c|c|c|c|c|}
\hline \multirow[t]{2}{*}{ Variable } & \multicolumn{2}{|c|}{3 Months } & \multicolumn{2}{|c|}{6 Months } & \multirow[t]{2}{*}{$f$} & \multirow[t]{2}{*}{$p$} \\
\hline & $M$ & $S D$ & M & $S D$ & & \\
\hline \multicolumn{7}{|l|}{ Outcome variables } \\
\hline Body mass index & 33.81 & 4.92 & 34.02 & 5.33 & 0.47 & .630 \\
\hline Binge eating & 12.33 & 7.90 & 14.67 & 12.40 & 1.97 & .159 \\
\hline Eating psychopathology & 2.17 & 0.90 & 2.33 & 1.08 & 0.22 & .818 \\
\hline \multicolumn{7}{|l|}{ Process variables } \\
\hline Psychological inflexibility & 26.69 & 8.65 & 25.31 & 10.13 & 0.59 & .561 \\
\hline Body image cognitive fusion & 30.27 & 15.21 & 30.33 & 17.85 & 0.16 & .852 \\
\hline Engaged with valued living & 28.57 & 6.38 & 25.36 & 6.71 & 0.33 & .721 \\
\hline External shame & 23.69 & 18.05 & 24.13 & 17.90 & 0.80 & .460 \\
\hline Inadequate-self & 16.20 & 8.36 & 15.73 & 8.30 & 0.49 & .619 \\
\hline Hated-self & 3.80 & 3.78 & 4.07 & 4.00 & 0.13 & .880 \\
\hline Reassured-self & 16.43 & 8.21 & 16.36 & 8.34 & 0.50 & .614 \\
\hline Self-judgement & 8.18 & 2.75 & 8.61 & 3.13 & 0.64 & .535 \\
\hline Self-compassion & 8.80 & 2.25 & 8.54 & 2.59 & 0.32 & .727 \\
\hline \multicolumn{7}{|l|}{ Mindfulness } \\
\hline Act awareness & 9.31 & 1.92 & 8.63 & 2.55 & 1.30 & .288 \\
\hline Non-judge & 9.81 & 1.76 & 9.69 & 2.60 & 1.28 & .293 \\
\hline Non-react & 8.93 & 2.38 & 8.81 & 1.57 & 0.66 & .525 \\
\hline Total & 46.81 & 8.16 & 45.69 & 8.64 & 0.36 & .702 \\
\hline
\end{tabular}

$S D$ : standard deviation.

comparing the results from post-intervention, 3- and 6-month follow-up. The results are presented in Table 2, and they suggest there are no significant differences between the three time points. Pairwise comparisons' post hoc analyses confirm these results, indicating that post-intervention therapeutic gains were maintained at 3- and 6-month follow-up in all variables.

\section{Mechanisms of change: processes underlying BEfree}

To explore possible mediators of the effect of the intervention on the outcomes, we conducted twocondition within-subjects' mediation analyses, with each mediator tested separately in the model.

Binge eating. The results showed significant indirect effects of the intervention on binge eating. Specifically, we found that psychological inflexibility $(B=-3.30$, BootSE $=1.34,95 \% \mathrm{CI}(-6.40$ to
$-1.04)$ ), body image cognitive fusion $(B=-5.14$, BootSE $=1.41,95 \%$ CI $(-8.35$ to -2.71$))$ and engaged with valued living $(B=-2.89$, BootSE $=1.18,95 \%$ CI $(-5.66$ to -0.90$))$ significantly mediated the effect of intervention on binge eating. Additionally, the changes in external shame $(B=-3.39$, BootSE $=1.36,95 \%$ CI $(-6.98$ to $-1.30))$, inadequate-self $(B=-2.77, \operatorname{BootSE}=1.29$, 95\% CI $(-6.08$ to -0.85$))$, hated-self $(B=-2.85$, BootSE $=1.26,95 \%$ CI $(-5.67$ to -0.74$))$, reassured-self $(B=-1.61$, BootSE $=1.03,95 \%$ CI $(-4.26$ to -0.08$))$, self-judgement $(B=-5.54$, BootSE $=1.72,95 \% \mathrm{CI}(-9.92$ to -3.00$))$ and selfcompassion $(B=-1.68$, BootSE $=1.16,95 \%$ CI $(-5.01$ to -0.17$))$ mediated the decrease in binge eating from pre- to post-intervention.

Eating psychopathology. The results showed that only general psychological inflexibility $(B=-0.23$, BootSE $=0.13,95 \%$ CI $(-0.54$ to -0.02$))$ and the non-reacting facet of mindfulness $(B=-0.25$, 
BootSE $=0.16,95 \%$ CI $(-0.08$ to -0.02$))$ significantly mediated the decrease in eating psychopathology as a result of the intervention.

In sum, several psychological processes targeted by BEfree mediated the effect of the intervention on the outcome measures, particularly on binge eating severity. However, mindfulness was not a significant mediator of the change in the outcomes, with the exception of the facet non-reacting that mediated the changes in eating psychopathology.

\section{Discussion}

There is growing evidence for the efficacy of psychological approaches focused on the development of mindfulness (Kristeller and Wolever, 2010), self-compassion (Kelly and Carter, 2015; Kelly et al., 2014) and the promotion of action congruent with personal values (Masuda et al., 2014) for eating psychopathology and BED. Nevertheless, although there is a recent interest in integrating these approaches in complimentary interventions for medical condition (Skinta et al., 2015), this was yet to be accomplished for BED.

This study explored the efficacy of a psychological group intervention for BED that integrates psychoeducation, mindfulness, compassion and value-based action, in a sample of 31 overweight and obese women. The results suggested that BEfree was able to diminish binge eating symptoms. In fact, after the intervention, none of the participants met criteria for a BED diagnosis, according to the EDE interview conducted by experienced clinical psychologists. Additionally, after the intervention, participants presented lower levels of eating psychopathology. Although BEfree was not developed to target weight itself, there was also a decrease in BMI. Regarding the psychological processes that BEfree was designed to tackle, there was a medium-to-large effect in the reduction in external shame, psychological inflexibility, body image cognitive fusion and self-criticism (both the most toxic forms - inadequate- and hated-self - and the more general self-judgemental attitude towards oneself). The results also showed that BEfree had medium-to-large effects in increasing valued actions, self-compassion and in the ability to act with awareness, non-judge and nonreact (mindfulness facets). Nevertheless, the results from mindfulness should be interpreted with caution, as the internal consistencies of FFMQ did not reach recommended values at baseline. There is, in fact, an ongoing discussion on the limitations of measuring mindfulness. Particularly, FFMQ's factor structure seems to vary depending on whether respondents are meditators or nonmeditators (Baer et al., 2008). Indeed, it is suggested that non-meditators might interpret items related to paying attention as undesirable awareness of intruding internal experiences (Grossman, 2011). Interestingly, when calculating the internal consistency of FFMQ at post-intervention, Cronbach's alphas yielded higher reliability (.49 for observing facet, .92 for describing, .72 for acting with awareness, .74 for non-judging, .64 for non-reacting and .82 for total scale), which seems to corroborate the suggestion that the items may be differently interpreted by individuals with different degrees of meditation experience.

We have also conducted repeated measures ANOVAs in order to explore whether the results obtained from pre- to post-intervention were maintained at 3- and 6-month follow-up. In fact, the results showed that the effectiveness of BEfree was maintained beyond the period of the programme, up to 6 months. The results showed that there were no significant differences from post-intervention to 3 and 6 months after the intervention, neither in the outcome measures nor in the psychological processes promoted by BEfree. In spite of these promising results, a careful interpretation is advised given the number of dropouts.

In addition to studying the efficacy of BEfree, we set out to explore which psychological processes promoted by the intervention led to the changes in the outcome measures. In fact, although it is crucial to develop empirically based psychological interventions for $\mathrm{BED}$, it is not less important to explore the mechanisms through which therapeutic change occurs (Kazdin, 2007; McCracken and Gutiérrez-Martínez, 2011; Murphy et al., 2009). The results showed that the decrease in binge eating severity from pre- to post-intervention was mediated by the diminishing of external 
shame and self-criticism. This is in line with previous research which suggested that shame and self-criticism are pervasive processes that maintain binge eating symptomatology (Duarte et al., 2014, 2015b; Gilbert, 2002; Goss and Allan, 2009; Goss and Gilbert, 2002). Our results suggest that diminishing shame and selfcriticism is a crucial goal when targeting binge eating. Previous research suggested that one way of decreasing shame and self-criticism is by promoting a more self-compassionate and self-reassuring stance towards the self (Gilbert, $2005,2009,2010)$. In accordance with this, our results also suggested that the effectiveness of BEfree in diminishing binge eating was mediated by an increase in self-compassion and selfreassurance. One way of making sense of the role of self-compassion is through the affect regulation systems. The self-compassionate exercises throughout BEfree might have helped to develop a positive soothing system that activates feelings of caring and warmth towards the self (e.g. Hermanto and Zuroff, 2016). On the other hand, this counteracts and helps to deactivate the threat-focused system that is responsible for negative emotions such as shame and self-criticism (e.g. Gilbert, 2005; Gilbert and Procter, 2006). Additionally, the results also suggested that diminishing psychological inflexibility, and specifically decreasing the entanglement with internal experiences focused on body image (i.e. body image cognitive fusion), as well as promoting action that is congruent with personal values, are important therapeutic goals when targeting BED. In fact, our results showed that the changes in psychological inflexibility, body image cognitive fusion and engagement with value-based action mediated the changes in binge eating severity. Recent studies highlighted the pervasive role of psychological inflexibility in disordered eating (Masuda et al., 2010, 2011), and only one study, to our knowledge, has suggested the pervasive role of body image cognitive fusion in the maintenance of binge eating severity (Duarte et al., 2015b). Additionally, although some interventions aimed at promoting value-based action yielded beneficial results for binge eating (Masuda et al., 2014), the specific role of value-based action in therapy for BED has never been empirically shown. To our knowledge, this study is the first to do so. Indeed, the mediational role of psychological inflexibility, body image cognitive fusion and engagement with valued living corroborates theoretical literature which suggests that binge eating is a way of controlling unwanted internal experiences (in this case, content related to one's body image), at the expense of living a valued life (Lillis et al., 2011). By promoting a more flexible and disentangled way of relating to personal thoughts and emotions related to body image, as well as by promoting an action guided by personal values, despite negative internal experiences, BEfree was effective in diminishing binge eating severity. Contrarily to our hypothesis, mindfulness did not mediate the changes in binge eating. Two possible explanations might be raised. One explanation is that mindfulness practices between sessions were not as intensively promoted as it could have been. Another possible explanation is the aforementioned difficulties in measuring mindfulness, and specifically the low internal consistencies of FFMQ in this study, particularly at baseline.

Regarding eating psychopathology, the changes were only mediated by psychological inflexibility and the non-reacting facet of mindfulness, even though this result for mindfulness should be viewed with caution. Nevertheless, these results show that almost none of the psychological processes (except psychological inflexibility) promoted by BEfree mediated the changes in eating psychopathology. In fact, BEfree was designed to target specifically binge eating behaviours, whereas EDE interview (which was used to assess eating psychopathology) was developed to assess disordered eating attitudes and behaviours in general. Since BED was just recently recognized as a specific eating disorder, there are not many items in EDE that focus on BED symptomatology, specifically in obesity. The fact that reductions in psychological processes such as external shame, self-criticism and body image cognitive fusion did not mediate the changes in eating psychopathology 
might suggest that in our sample these processes are highly related to binge eating, and that eating psychopathology as measured by EDE does not properly reflect binge eating symptomatology. Therefore, the changes in these variables did not yield the changes in eating psychopathology. Interestingly, psychological inflexibility, which is a general measure of unwillingness to have difficult thoughts (not exclusively related to binge eating), mediated the changes in eating psychopathology, which seems to be aligned with this hypothesis that eating psychopathology assessed by EDE encompasses other aspects of disordered eating attitudes and behaviours that might not reflect what binge eaters tend to experience.

In sum, BEfree seems to be an effective programme for diminishing binge eating and overall eating psychopathology, with positive results maintained up to 6 months after the end of the intervention. Additionally, the reductions in binge eating were mediated by the decreases in external shame, self-criticism, psychological inflexibility and body image cognitive fusion, as well as increases in self-compassion and valuebased action. These results seem to provide evidence for the efficacy of BEfree and it corroborates the effectiveness of integrating different yet complimentary psychological approaches when targeting BED.

Several limitations should be taken into consideration when interpreting our results. First, the relatively small sample limits the generalizability of these results to the wider population. These results should be replicated in a larger sample. Additionally, the sample comprised only women, which does not allow us to extrapolate these results to a sample of men. In fact, the relative impact of the processes promoted by BEfree might be different in a sample of males. Not less important is the fact that this study did not compare the results with a control group, which calls for caution when interpreting these results. Finally, as stated throughout this study, it is important to consider the low reliability of FFMQ when interpreting all results related to mindfulness. This study used a shorter 15-item version of FFMQ, in which each subscale was composed of only 3 items. Future studies that aim to explore the impact of BEfree in the different facets of mindfulness should use the longer 39-item version.

Nonetheless, this study is the first to integrate complimentary components of different but theoretically coherent approaches in a psychological programme for women with BED and obesity. This study is also a valid contribution as it provides data for the efficacy of BEfree up to a follow-up period of 6 months. Finally, we explored the mediational role of several psychological processes promoted by BEfree in the reduction in binge eating severity and eating psychopathology, therefore contributing for a better understanding of which processes should be targeted in BED therapy.

\section{Declaration of conflicting interests}

The author(s) declared no potential conflicts of interest with respect to the research, authorship, and/or publication of this article.

\section{Funding}

The author(s) disclosed receipt of the following financial support for the research, authorship, and/or publication of this article: This research was supported by Portuguese Foundation for Science and Technology with the R\&D Grant (PTDC/MHC-PCL/4923/2012).

\section{References}

Ahmadpanah M, Paghale SJ, Bakhtyari A, et al. (2014) Effects of psychotherapy in combination with pharmacotherapy, when compared to pharmacotherapy only on blood pressure, depression, and anxiety in female patients with hypertension. Journal of Health Psychology 21(7): 1216-1227.

Albertson ER, Neff KD and Dill-Shackleford KE (2015) Self-compassion and body dissatisfaction in women: A randomized controlled trial of a brief meditation intervention. Mindfulness 6(3): 444-454.

American Psychiatric Association (APA) (2013) Diagnostic and Statistical Manual of Mental Disorders: DSM-5. Arlington, VA: American Psychiatric Publishing.

Baer RA, Hopkins J, Krietemeyer J, et al. (2006) Using self-report assessment methods to explore facets of mindfulness. Assessment 13(1): 27-45. 
Baer RA, Smith GT, Lykins E, et al. (2008) Construct validity of the five facet mindfulness questionnaire in meditating and nonmeditating samples. Assessment 15(3): 329-342.

Bond FW, Hayes SC, Baer RA, et al. (2011) Preliminary psychometric properties of the Acceptance and Action Questionniare - II: A revised measure of psychological flexibility and experiential avoidance. Behavior Therapy 42: 676-688.

Bulik CM, Sullivan PF and Kendler KS (2002) Medical and psychiatric morbidity in obese women with and without binge eating. International Journal of Eating Disorders 32(1): 72-78.

Castilho P, Pinto-Gouveia J and Duarte J (2015) Evaluating the multifactor structure of the long and short versions of the self-compassion scale in a clinical sample. Journal of Clinical Psychology 71(9): 856-870.

Cohen J (1988) Statistical Power Analysis for the Behavioral Sciences (2nd edn). Hillsdale, NJ: Lawrence Erlbaum.

Costa J, Marôco J, Pinto-Gouveia J, et al. (2015) Validation of the psychometric properties of the Self-Compassion Scale. Testing the factorial validity and factorial invariance of the measure among borderline personality disorder, anxiety disorder, eating disorder and general populations. Clinical Psychology \& Psychotherapy 23: 460-468.

Dingemans AE, Bruna MJ and Van Furth EF (2002) Binge eating disorder: A review. International Journal of Obesity 26(3): 299-307.

Duarte C, Ferreira C, Trindade IA, et al. (2015a) Body image and college women's quality of life: The importance of being self-compassionate. Journal of Health Psychology 20(6): 754-764.

Duarte C, Pinto-Gouveia J and Ferreira C (2014) Escaping from body image shame and harsh self-criticism: Exploration of underlying mechanisms of binge eating. Eating Behaviors 15(4): 638-643.

Duarte C, Pinto-Gouveia J and Ferreira C (2015b) Ashamed and fused with body image and eating: Binge eating as an avoidance strategy. Clinical Psychology \& Psychotherapy. Epub ahead of print 21 December. DOI: 10.1002/ cpp.1996.

Dunne S, Sheffield D and Chilcot J (2016) Brief report: Self-compassion, physical health and the mediating role of health-promoting behaviours. Journal of Health Psychology. Epub ahead of print 26 April. DOI: 10.1177/1359105316643377.

Fairburn CG, Cooper Z and O'Connor M (2008) Eating disorder examination (16.0D). In: Fairburn CG (ed.) Cognitive Behavior Therapy and Eating Disorders. New York: Guilford Press, pp. 265-308.

Ferreira C, Trindade IA, Duarte C, etal. (2015) Getting entangled with body image: Development and validation of a new measure. Psychology and Psychotherapy: Theory, Research and Practice 88(3): 304-316.

Gilbert P (2002) Body shame: A biopsychosocial conceptualisation and overview, with treatment implications. In: Gilbert P and Miles J (eds) Body Shame: Conceptualisation, Research and Treatment. New York: Brunner Routledge, pp. 3-54.

Gilbert P (2005) Compassion: Conceptualisations, Research and Use in Psychotherapy. New York: Routledge.

Gilbert P (2009) Introducing compassion-focused therapy. Advances in Psychiatric Treatment 15(3): 199-208.

Gilbert P (2010) The Compassionate Mind: A New Approach to Life's Challenges. Oakland, CA: New Harbinger Publications.

Gilbert P and Procter S (2006) Compassionate mind training for people with high shame and selfcriticism: Overview and pilot study of a group therapy approach. Clinical Psychology \& Psychotherapy 13(6): 353-379.

Gilbert P, Clarke M, Hempel S, et al. (2004) Criticizing and reassuring oneself: An exploration of forms, styles and reasons in female students. British Journal of Clinical Psychology 43(1): 31-50.

Gillanders DT, Bolderston H, Bond FW, et al. (2014) The development and initial validation of the Cognitive Fusion Questionnaire. Behavior Therapy 45(1): 83-101.

Gormally J, Black S, Daston S, et al. (1982) The assessment of binge eating severity among obese persons. Addictive Behaviors 7(1): 47-55.

Goss K and Allan S (2009) Shame, pride and eating disorders. Clinical Psychology \& Psychotherapy 16: 303-316.

Goss K and Gilbert P (2002) Eating disorders, shame and pride: A cognitive-behavioural functional analysis. In: Gilbert P and Miles J (eds) Body Shame Conceptualisation, Research and Treatment. New York: Brunner Routledge, pp. 219-255. 
Goss K, Gilbert P and Allan S (1994) An exploration of shame measures - I: The Other as Shamer Scale. Personality and Individual Differences 17(5): 713-717.

Grossman P (2011). Defining mindfulness by how poorly I think I pay attention during everyday awareness and other intractable problems for psychology's (re)invention of mindfulness: Comment on Brown et al. (2011). Psychological Assessment 23(4): 1034-1040. DOI: 10.1037/a0022713.

Hayes SC, Luoma JB, Bond FW, et al. (2006) Acceptance and commitment therapy: Model, processes and outcomes. Behaviour Research and Therapy 44(1): 1-25.

Hermanto N and Zuroff DC (2016) The social mentality theory of self-compassion and self-reassurance: The interactive effect of care-seeking and caregiving. Journal of Social Psychology 6: $1-13$.

Juarascio A, Forman E and Herbert J (2010) Acceptance and commitment therapy versus cognitive therapy for the treatment of comorbid eating pathology. Behavior Modification 34(2): 175-190.

Juarascio A, Shaw J, Forman E, et al. (2013) Acceptance and commitment therapy as a novel treatment for eating disorders: An initial test of efficacy and mediation. Behavior Modification 37(4): 459-489.

Kazdin AE (2007) Mediators and mechanisms of change in psychotherapy research. Annual Review of Clinical Psychology 3: 1-27.

Kelly AC and Carter JC (2015) Self-compassion training for binge eating disorder: A pilot randomized controlled trial. Psychology and Psychotherapy: Theory, Research and Practice 88(3): 285-303.

Kelly AC, Vimalakanthan K and Miller K (2014) Self-compassion moderates the relationship between body mass index and both eating disorder pathology and body image flexibility. Body Image 11(4): 446-453.

Kristeller J and Wolever R (2010) Mindfulnessbased eating awareness training for treating binge eating disorder: The conceptual foundation. Eating Disorders 19(1): 49-61.

Leaviss J and Uttley L (2015) Psychotherapeutic benefits of compassion-focused therapy: An early systematic review. Psychological Medicine 45(5): 927-945.

Leehr EJ, Krohmer K, Schag K, et al. (2015) Emotion regulation model in binge eating disorder and obesity - A systematic review. Neuroscience and Biobehavioral Reviews 49: 125-134.

Lillis J and Kendra K (2014) Acceptance and Commitment Therapy for weight control: Model, evidence, and future directions. Journal of Contextual Behavioral Science 3(1): 1-7.

Lillis J, Hayes SC and Levin ME (2011) Binge eating and weight control: The role of experiential avoidance. Behavior Modification 5(3): 252-264.

López A, Sanderman R, Smink A, et al. (2015) A reconsideration of the Self-Compassion Scale's total score: Self-compassion versus self-criticism. PloS ONE 10(7): e0132940.

McCracken LM and Gutiérrez-Martínez O (2011) Processes of change in psychological flexibility in an interdisciplinary group-based treatment for chronic pain based on Acceptance and Commitment Therapy. Behaviour Research and Therapy 49(4): 267-274.

Marcus MD, Wing RR and Lamparski DM (1985) Binge eating and dietary restraint in obese patients. Addictive Behaviors 10(2): 163-168.

Marta-Simões J, Ferreira C and Mendes AL (2016) Self-compassion: An adaptive link between early memories and women's quality of life. Journal of Health Psychology. Epub ahead of print 10 July. DOI: 10.1177/1359105316656771.

Masuda A, Boone M and Timko C (2011) The role of psychological flexibility in the relationship between self-concealment and disordered eating symptoms. Eating Behaviors 12: 131-135.

Masuda A, Hill ML, Melcher H, et al. (2014) Acceptance and commitment therapy for women diagnosed with binge eating disorder: A case-series study (Paper 89). Available at: http://scholarworks.gsu.edu/psych_facpub/89

Masuda A, Price M, Anderson PL, et al. (2010) Disordered eating-related cognition and psychological flexibility as predictors of psychological health among college students. Behavior Modification 34(1): 3-15.

Matos M, Pinto-Gouveia J, Gilbert P, et al. (2015) The Other As Shamer Scale-2: Development and validation of a short version of a measure of external shame. Personality and Individual Differences 74: 6-11.

Montoya AK and Hayes AF (2016) Two condition within-participant statistical mediation analysis: A path-analytic framework. Psychological Methods. Epub ahead of print 30 June. DOI: $10.1037 /$ met0000086. 
Murphy R, Cooper Z, Hollon SD, et al. (2009) How do psychological treatments work? Investigating mediators of change. Behaviour Research and Therapy 47(1): 1-5.

Mussell M, Mitchell J, de Zwaan M, et al. (1996) Clinical characteristics associated with binge eating in obese females: A descriptive study. International Journal of Obesity and Related Metabolic Disorders 20: 324-331.

Neff KD (2003) The development and validation of a scale to measure self-compassion. Self and Identity 2(3): 223-250.

Neff KD (2016) The Self-Compassion Scale is a valid and theoretically coherent measure of selfcompassion. Mindfulness 7: 264-274.

O'Leary K, O'Neill S and Dockray S (2015) A systematic review of the effects of mindfulness interventions on cortisol. Journal of Health Psychology 21(9): 2108-2121.

Picot AK and Lilenfeld LR (2003) The relationship among binge severity, personality psychopathology, and body mass index. International Journal of Eating Disorders 34(1): 98-107.

Pinto-Gouveia J, Gregório S, Dinis A, et al. (2012) Experiential avoidance in clinical and nonclinical samples: AAQ-II Portuguese version. International Journal of Psychology and Psychological Therapy 12(2): 139-156.

Skinta MD, Lezama M, Wells G, et al. (2015) Acceptance and compassion-based group therapy to reduce HIV stigma. Cognitive and Behavioral Practice 22(4): 481-490.
Sobik L, Hutchison K and Craighead L (2005) Cueelicited craving for food: A fresh approach to the study of binge eating. Appetite 44: 253-261.

Spitzer RL, Yanovski S, Wadden T, et al. (1993) Binge eating disorder: Its further validation in a multisite study. International Journal of Eating Disorders 13(2): 137-153.

Sysko R, Devlin MJ, Walsh BT, et al. (2007) Satiety and test meal intake among women with binge eating disorder. International Journal of Eating Disorders 40: 554-561.

Telch CF and Stice E (1998) Psychiatric comorbidity in women with binge eating disorder: Prevalence rates from a non-treatment-seeking sample. Journal of Consulting and Clinical Psychology 66(5): 768-776.

Trindade IA, Ferreira C, Pinto-Gouveia J, et al. (2015) Clarity of personal values and committed action: Development of a shorter engaged living scale. Journal of Psychopathology and Behavioral Assessment 1-8.

Trompetter HR, ten Klooster PM, Schreurs KM, et al. (2013) Measuring values and committed action with the Engaged Living Scale (ELS): Psychometric evaluation in a nonclinical sample and a chronic pain sample. Psychological Assessment 25(4): 1235-1246.

Xu W, Oei TP, Liu X, et al. (2014) The moderating and mediating roles of self-acceptance and tolerance to others in the relationship between mindfulness and subjective well-being. Journal of Health Psychology 21(7): 1446-1456. 